\title{
Evaluating Depth Perception of Photorealistic Mixed Reality Visualizations for Occluded Objects in Outdoor Environments
}

\author{
Arindam Dey* $\quad$ Andrew Cunningham ${ }^{\dagger} \quad$ Christian Sandor $^{\ddagger}$ \\ Wearable Computer Laboratory, School of Computer and Information Science, University of South Australia
}

\begin{abstract}
Enabling users to accurately perceive the correct depth of occluded objects is one of the major challenges in user interfaces for Mixed Reality (MR). In this paper, we present an evaluation of depth perception in handheld outdoor mixed reality environment in far-field distances through two photorealistic visualizations of occluded objects (X-ray and Melt) in the presence and absence of a depth cue.
\end{abstract}

Index Terms: H.5.1 [Information Interfaces and Presentation]: Multimedia Information Systems-Artificial, Augmented, Virtual, and Mixed Realities H.5.2 [User Interfaces]: Ergonomics, Evaluation / Methodology—Screen Design, Style Guides

\section{InTROduction AND RELATEd WORK}

Properly visualizing occluded objects is a challenging task in mixed reality (MR). Many interactive MR visualizations have been developed to display occluded objects in outdoor environments, such as our previously described Melt visualization [3] to display occluded objects by virtually melting the occluder, or our outdoor $X$-ray visualization [1], where the edges of occluding objects were highlighted in the video image to provide a realistic sense of occlusion. We categorize these techniques as photorealistic visualizations, as they attempt to realistically portray the occluded and occluder objects, creating a natural blend between the real and virtual space of the outdoor MR environment. Many studies have experimented the depth estimation in MR environments such as Livingston et al. [2] compared distance estimation aided with two different depth cues-gridpoint and tramline-in both an indoor and an outdoor environment using HMDs. Contrary to the results in indoor environments, they found that distance is overestimated in outdoor environments in medium to far-field distances. We took inspiration for our research from this work, however the main contribution of our experiment is the evaluation of photorealistic visualizations on depth perception in far-field outdoor MR environments purposefully using a handheld display, as it is not widely experimented in MR so far.

\section{EXPERIMENT}

We have investigated photorealistic visualizations for viewing occluded objects in outdoor MR using a handheld display. This mixed design experiment, involving twenty ( 2 female, 18 male) participants, was based on five independent variables as shown in Table 1. All variables were within subject except the Depth Cue and Participant group.

The experimental conditions (Figure 1) were presented on a 7" handheld display with $640 \times 480$ resolution attached to a laptop mounted on a tripod facing a building at 29 meters away. Participants had to report the egocentric distance of a $3 \times 3$ meter rendered

*e-mail: Arindam.Dey@postgrads.unisa.edu.au

†e-mail: Andrew.Cunningham@postgrads.unisa.edu.au

†e-mail: christian@sandor.com
Table 1: Independent variables.

\begin{tabular}{llr}
\hline Name & No. of Levels & Description \\
\hline Visualization & 2 & X-ray, Melt \\
Depth Cue & 2 & On, Off \\
Distance & 5 & $69.7 \mathrm{~m}, 82.5 \mathrm{~m}, 95.1 \mathrm{~m}$, \\
& & $104.6 \mathrm{~m}, 117.0 \mathrm{~m}$ \\
Participant Group & 2 & A, B \\
Trial & 30 & T1 . T30 \\
\hline
\end{tabular}

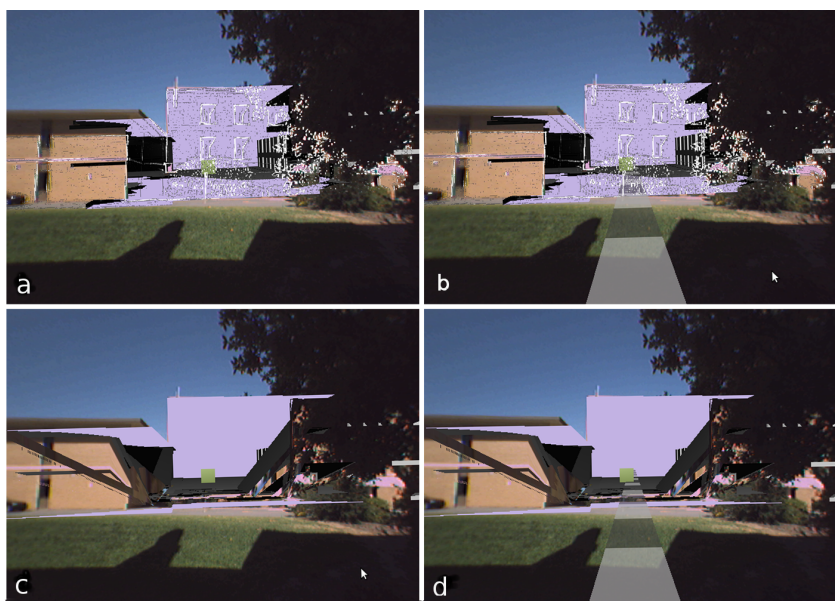

Figure 1: Four different experimental conditions: (a) X-ray + Cue-off, (b) X-ray + Cue-on, (c) Melt + Cue-off, (d) Melt + Cue-on.

green cube. Each participant experienced both visualizations (Melt and X-ray) across all five distances, achieving ten unique treatments per participant. Each treatment was repeated in a randomized order three times, resulting in 30 trials per participant. The graphical depth cue was treated as a between-subjects condition, with ten participants experiencing the graphical cue (cue-on) and ten participants without a graphical cue (cue-off). Our graphical cue is rendered as a semi-transparent segmented path originating from the user in the direction of the target. Each segment is 10 meters in length and alternates between black and white in color. The total experiment resulted in 2 (visualizations) $\times 5$ (distances) $\times 2$ $($ graphical cues $) \times 10$ (participants $) \times 3$ (repetitions $)=600$ data points. Participants verbally reported the perceived distance $(P D)$ to the experimenter who recorded the PD and response time.

\section{Results}

Three dependant variables were derived from the responses of the participants: Signed Error (SE), Accuracy, and Response Time. SE and Accuracy was individually measured as functions of actual distance and perceived distance.

Signed Error: Distance was mostly underestimated in all conditions (Figure 2). The graphical cue significantly reduced error 


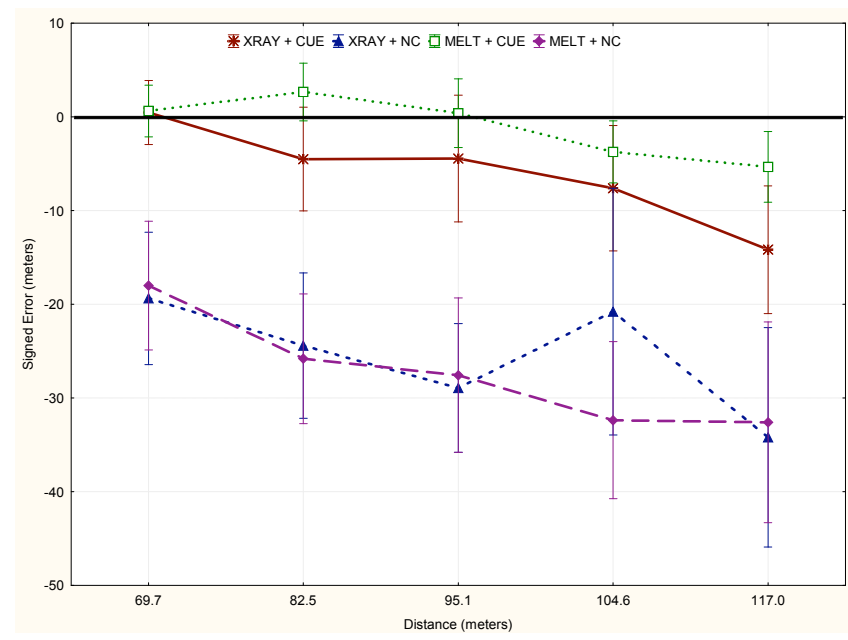

Figure 2: Our experiment revealed a consistent underestimation of distances in an outdoor MR environment. The thick line at 0.0 denotes veridical perception.

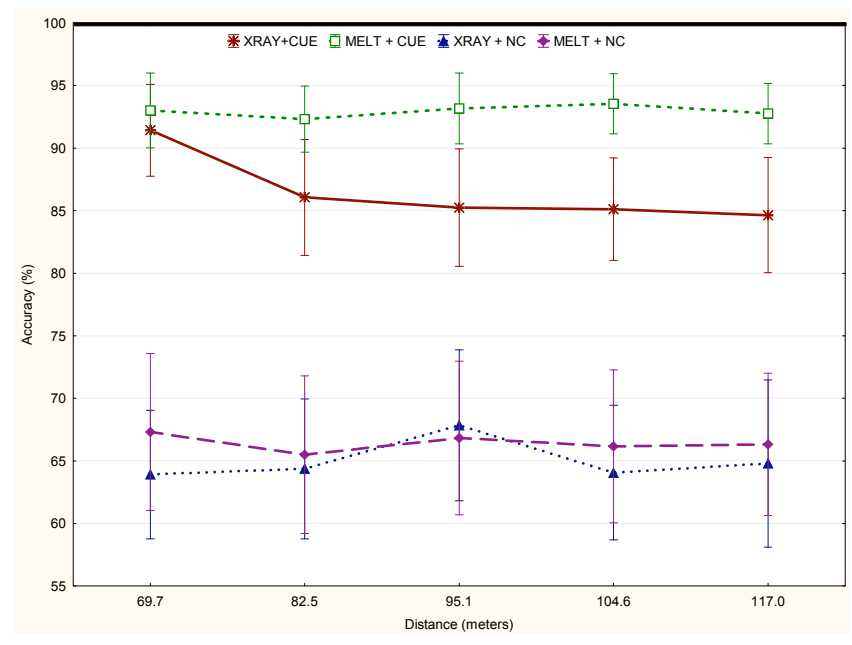

Figure 3: Accuracy shows an expected and significant improvement with the graphical cue conditions across both visualizations.

in both Melt and X-ray $(\mathrm{p}<0.0001)$. We also observed a main effect of distance on the signed error for both Melt $(F(4,295)=2.4389$, $\mathrm{p}<0.05)$ and $\mathrm{X}$-ray $(\mathrm{F}(4,295)=3.1249, \mathrm{p}=0.016)$.

Accuracy: Our graphical depth cue aided participants to perceive distance more accurately than without the cue. After running a one-tailed t-test we found that both X-ray+cue-on and Melt+cueon was significantly $(\mathrm{p}<0.001)$ more accurate than $\mathrm{X}$-ray+cueoff and Melt+cue-off respectively. Interestingly, we found that Melt+cue-on was significantly $(\mathrm{p}<0.001)$ more accurate than $\mathrm{X}$ ray+cue-on, and with increasing distance and the graphical cue, the accuracy of Melt stayed constant, while X-ray lost accuracy (Figure 3 ). We attribute this result to the visual noise created by our X-ray.

Response Time: In this experiment we found that the graphical cue caused a delayed response from participants. This makes sense, since participants had to count the segments of the cue to accurately interpret the distance. The mean response time of the cue-on was significantly $(\mathrm{p}<0.001)$ higher than cue-off condition for both $\mathrm{X}$ ray and Melt visualizations (see Figure 4 ). In the case of the cue-on condition Melt was significantly $(\mathrm{p}<0.001)$ faster then $\mathrm{X}$-ray. We predict that the low visibility due to the edge overlay of our X-ray

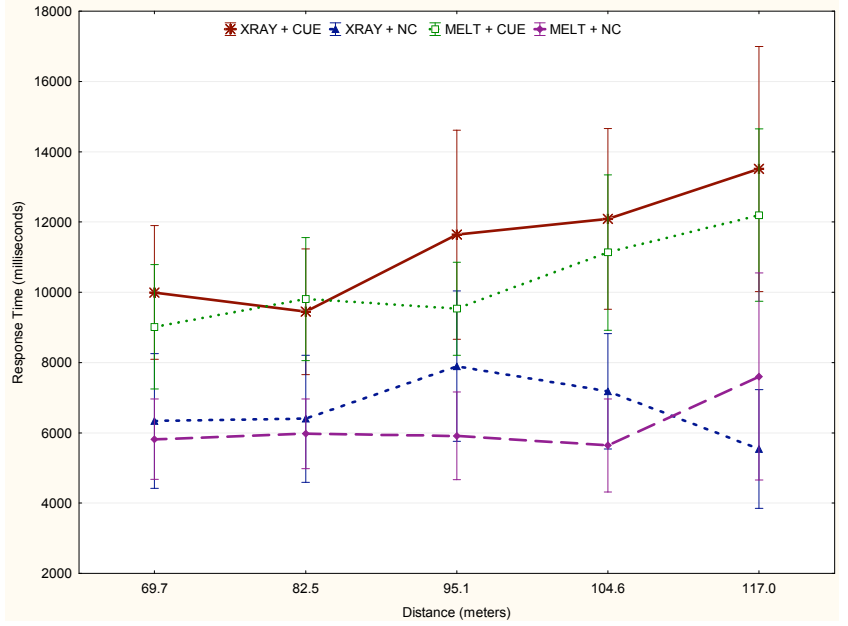

Figure 4: Response time at different distances in different experimental conditions.

visualization made it harder to count the segments of the graphical cue.

\section{Discussion}

The most important result of this experiment is the observation of an underestimation of the distance of occluded objects in outdoor environments which seemingly contradicts the findings of Livingston et al. [2]. We can imagine four possible reasons for this contradiction: (a) use of a handheld display as it provides a lesser level of immersion than head mounted displays, (b) use of a video see-through setup, (c) use of our MR system which is more akin to a virtual reality system than Livingston et al's, and (d) the dense edge overlay of our X-ray visualization. Graphical depth cue added accuracy but increased response time as well. It is because participants had to count the segments of the depth cue before responding. Melt provided a clear scene and made the target object completely visible, which resulted in significantly positive results in favor of Melt over $\mathrm{X}$-ray, both in terms of accuracy and response time.

\section{FUtURE WORK AND CONCLUSION}

This experiment was one of the first efforts to evaluate photorealistic visualizations for distance perception in MR using handheld displays. We observed distance underestimation using handheld displays in an outdoor environment. We plan to verify the validity of our results with a more extensive user study and aim to contrast this result with a HMD. In this experiment we had to rigidly mount the handheld display on a tripod to overcome the adverse effect of tracking, we plan use the handheld display as "hand held" with an improved tracking in a future study. We also plan to compare various depth cues available for MR environments.

\section{REFERENCES}

[1] B. Avery, C. Sandor, and B. H. Thomas. Improving spatial perception for augmented reality $\mathrm{x}$-ray vision. In $V R$ '09: Proceedings of the IEEE Conference on Virtual Reality, pages 79-82. IEEE, 2009.

[2] M. A. Livingston, Z. Ai, J. Swan, and H. S. Smallman. Indoor vs. outdoor depth perception for mobile augmented reality. In $V R$ '09: Proceedings of the IEEE Conference on Virtual Reality, pages 55-62, Washington, DC, USA, 2009. IEEE Computer Society.

[3] C. Sandor, A. Cunningham, U. Eck, D. Urquhart, G. Jarvis, A. Dey, S. Barbier, M. Marner, and S. Rhee. Egocentric space-distorting visualizations for rapid environment exploration in mobile mixed reality. In VR 2010: Proceedings of IEEE Conference on Virtual Reality, 2009, to appear. 